\section{LA FENOMENOLOGÍA COMO ATAVISMO}

\author{
Iván Galán \\ Bergische Universität Wuppertal
}

\begin{abstract}
This paper deals with phenomenology as a result of phenomenological freedom in its possibility of understanding a phenomenon as wild phenomenon. From this perspective, we are enabled to transcend established symbolic systems and to pose the question on the existence of a sense which is immature, not-saturated and imperfect without exception.
\end{abstract}

KEY WORDS: Phenomenological freedom, wild sense, symbolical institution, perception, logical meaning.

\section{INTRODUCCIÓN}

El presente artículo pretende dar cuenta de algunos temas que, por su recurrencia e importancia, constituyen elementos centrales de la fenomenología desde sus albores. No he pretendido realizar, sin embargo, un retrato exhaustivo de la fenomenología desde la totalidad de sus temas. A este respecto cabe tan sólo decir que cualquier afán de exhaustividad debe ser inmediatamente postergado, pues la proliferación de temas y tratamientos en el ámbito de la fenomenología es, como se sabe, prácticamente inabarcable para la vista. Acometer una enumeración detallada de los mismos es, acaso, una tarea imposible.

Por lo que hace a aquellos temas que este artículo pone en avatar de colación, apenas sí podrá el lector encontrar esbozos y trazos hechos al desgaire, redactados muchas veces casi que a vuelapluma. Con ello no he intentado sino subvenir, de la mejor manera posible, a la petición de los editores de esta revista, a quien hago público mi infinito agradecimiento. El artículo debía ser acometido en registro literario, sin dar demasiado pábulo al academicismo.

El artículo presenta la siguiente articulación: en un primer momento, a modo de introducción, se planteará la pregunta por la vigencia de la fenomenología en tanto

\section{THE PHENOMENOLOGY AS AN ATAVISM}

RESUMEN: El artículo pretende mostrar la vigencia de la fenomenología como expresión de la libertad fenomenológica, como posibilidad siempre abierta de acceder al fenómeno en cuanto fenómeno puro, registro arquitectónico en el que, saltando por sobre los sistemas simbólicos instituidos, nos es permitido plantear en toda su radicalidad la pregunta por la existencia de un sentido siempre prematuro, no saturado y jamás clausurable.

PALABRAS CLAVE: Libertad fenomenológica, sentido salvaje, institución simbólica, percepción, significado lógico.

que fenomenologizar ${ }^{1}$, sometiendo a revisión el concepto de historia con el cual nos las vemos de cotidiano. En el siguiente epígrafe se intentará pergeñar en caracteres rápidos el doble movimiento del fenomenologizar: como epojé de un lado, y como reducción de otro; ambos elementos no son tratados en su valor sistemático, sino vistos en su carácter salvaje, como posibilidades de la humana libertad. En un último abordaremos un rápido retrato de la experiencia en clave fenomenológica, importando para ello ideas de diferentes autores.

Cumple decir que este artículo debe mucho en aspiración e inspiración a la fenomenología de Marc Richir, la cual nos ha cautivado, quizá, de manera definitiva, así como a las muchas conversaciones mantenidas con Pablo Posada Varela, a quien por su paciencia y generosidad extiendo mi gratitud.

\section{LA PERTINENCIA de LA FENOMENOLOGÍA}

Evaluar cuál sea el rol que a la fenomenología quepa asignarle en nuestros días no es tarea en absoluto fácil. Adoptando la figura de un trasgo juguetón y travieso la fenomenología no se deja encontrar dos veces en el mismo 
sitio; en la elasticidad de su concepto, en su heterodoxia para consigo misma, radica buena parte de su inagotabiliadad esencial. $Y$ es que, como es sabido, esta corriente de pensamiento ha seguido caminos en extremo dispares, difíciles a veces de conciliar y subsumir bajo un mismo marchamo ideológico. Tan sólo una cosa es segura: ese movimiento, en su difusión, en su heterogeneidad, en su marcha plural y tan poco unánime, ha experimentado en los últimos veinte años una revitalización incuestionable. Lejos de haberla eclipsado, el florecimiento de diversos movimientos filosóficos en Europa tras la Segunda Guerra Mundial como el existencialismo, el estructuralismo, la deconstrucción o la teoría crítica, no han venido sino a consignar la permeabilidad, la liquidez de principio que la caracteriza. Es precisamente en este marco de efervescencia en el cual surge y se inserta la pregunta por la vigencia de la fenomenología.

Mas esta pregunta, por muy atinada, por muy acuciante que su pertinencia histórica sea, sólo se deja responder desde la esencia misma de la fenomenología: desde el fenomenologizar como actividad de orden especial en la cultura. La validez de la fenomenología, su necesidad misma, son correlatos esenciales de su propia definición, siendo la fenomenología como es una institución simbólica que detenta un lugar especial entre todas las demás. Cabe decir que la necesidad del fenomenologizar no se deja, quizá, evaluar al tenor de razones puramente históricas. Ella quiere ser mucho más que un producto histórico, mucho más que el logro en saber científico obrado por la acumulación del trabajo conjunto de generaciones de investigadores $^{2}$. La necesidad del fenomenologizar no detrae su vigor de una determinada situación histórica, tampoco del estado general de las ciencias o de su posible utilidad para una sociedad determinada. La fenomenología se convalida a sí misma, la validez que quepa asignarle es, por su propia naturaleza, tributaria de su quehacer mismo, del fenomenologizar en cuanto tal. La fenomenología se interroga por el sentido del pensar y de ser de la fenomenalidad, $y$, aún siendo la pregunta a la que este texto debe su ocasión, con todo, una pregunta de orden histórico, una pregunta arrojada en un instante de la historia, no se deja abordar de manera convencional, pues la fenomenología no acepta, no puede aceptar, la jurisdicción de la historia (Historie) como última instancia simbólica por lo que hace a la determinación del sentido de ser de lo humano. La fenomenología incide sobre este punto, retoma la facticidad del devenir en su concretud fenomenológica y nos lanza por encima de la institución de la historia, proyectándonos hacia una dimensión de radical libertad humana, que no es otra que la dimensión de la libertad fenomenológica.

Todo fenómeno se hace pertinente a mi subjetividad como fenómeno de mundo; esto significa que el fenómeno se da siempre en una trenza de implicaciones intencionales o por relación a otros fenómenos, dando origen a la cohesión sin concepto $^{3}$ que caracteriza el campo fenoménico. Con otras palabras: todo fenómeno se halla abigarrado en la trascendencia de un mundo, adscrito a un horizonte sobre el cual, sin solución de continuidad, difracta. Mi carne corpórea (Leib), mi propio cuerpo, no admite excepción y se conforma como fenómeno individual en la contingencia de su fenomenalización a través de las sensaciones como fenómeno de mundo. Sensación, cuerpo, movimiento y percepción forman un tejido no desleible en el cual se acredita la irreducible disyunción del cuerpo en relación con el espacio y viceversa. El cuerpo, mi propia carne corpórea, forma parte de la cohesión sin concepto del mundo. Las sensaciones cinestésicas del movimiento corporal -así como las sensaciones de localización corporal (ubiestesias) $^{4}$ a través de las cuales se me da mi propia consistencia corpórea- están en intima correlación con en el entorno perceptivo. No hay sensaciones sin puesta en escena de esquematismo corporal, sin que los horizontes del cuerpo como fenómeno de mundo, coextensivos en su singularidad del resto de esencias sin concepto, estén dados en avanzada. Ambos fenómenos, sensación y cuerpo se sobrecruzan de manera irreducible, sin que podamos hacer nada para divorciarlos.

A tenor de lo dicho se puede establecer que el cuerpo funciona desde siempre como punto quiasmático entre mi subjetividad como intimidad de mundo y la cohesión sin concepto del mundo en la que él mismo, a través del esquematismo corporal, se inserta. Mi subjetividad, empero, se hace sutil al fenómeno en virtud de un esquematismo (de un reenvío logológico), que la "abrocha" o pone en relación con el mismo. El hombre, atornillado de una vez para siempre a un sentido sometido a constante fabricación y revisión queda deslocalizado como especie biológica y vinculado de una vez para siempre a la libertad que el fenómeno porta, a la libertad del fenómeno como fenómeno de mundo. En su serme pertinente pone el fenómeno de por medio una dimensión de sentido común, 
de sensus comunis, ingrediente irreducible de la libertad fenomenológica. Una particularidad de esta libertad radica en su carácter vinculante e intersubjetivo, pues se impone por igual a toda conciencia como un imperativo de la fenomenalización en cuanto tal. Con otras palabras: se trata de una libertad intermonádica, cuya universalidad y su necesidad son absolutamente comunicables en tanto en cuanto son coextensivas del sensus comunis como médium de fenomenalidad. A través de esta libertad los hombres se fenomenalizan para sí mismos y para los otros.

Este a priori humano de libertad deriva su vigor todo de la inclausurabilidad de principio propia al fenómeno como puro fenómeno, barrenando, vez a vez, la determinación del sentido de ser de lo humano operada desde la institución de la historia. La fenomenología pone, por consiguiente, a la vista una dimensión de lo histórico humano que se hace patente a la luz de un nuevo viso: como facticidad de un sentido humano operándose, como acontecimiento histórico o devenir (historia como Geschichte) de un sentido en constante fabricación y reelaboración. De este modo se puede hablar de una fundación simbólica de la historia y de la política, pues éstas, lejos de estar determinadas en su significatividad de una vez para siempre en el estricto marco de la institución, fundan simbólicamente en la libertad fenomenológica que dispensa el fenómeno como puro fenómeno, revelando en todo su dintorno lo humano desde su dimensión histórico-política.

La libertad que porta el fenómeno en su constitutiva irresolución determina lo humano en su faceta siempre incoativa como diacronía de una libertad inagotable o siempre haciéndose, y por lo tanto, en cierto modo absoluta. Está libertad del sentido haciéndose en su dimensión de sensus comunis apunta a la política ${ }^{5}$ acaso como índice de un inmarcesible fenomenológico: aquel de la libertad del hombre. Esta libertad fenomenológica que subyace a lo que aquí hemos dado en llamar fundación simbólica de la política y de la historia constituye un filamento esencial de la fenomenalidad, un reducto salvaje en el corazón del fenómeno toda vez que no se halla mediatizado por otras instituciones humanas.

Quizá quepa cifrar en punto a lo dicho en este primer epígrafe un primer aspecto de la vigencia imprescindible del quehacer fenomenológico. Frente a la coacción de la institución, que amenaza con anquilosar bajo la forma de un precipitado unívoco el sentido haciéndose de lo humano en su radical libertad de principio, se vuelve el fenomenologizar. En este sentido constituye el fenomenologizar el primer movimiento de la libertad humana. En el fenomenologizar y con él le es dado al ser humano, no ya en su calidad de especie biológica, sino como fenómeno de mundo cuyo sentido vale determinar, poner en solfa los valores que la cultura ha ido precipitando como baldones sobre la libertad que aquí hemos intentado definir.

\section{La fenomenología como ciencia salvaje}

La fenomenología se interroga por el sentido, mas no desde una presunta exterioridad o trascendencia indiferente que quisiere controlar desde fuera los parámetros que han de guiar las pesquisas. Que la fenomenología sea ante todo fenomenologizar significa que ella misma se hace aventura, apuesta su propio concepto al proceso, y no se reconoce de antemano en ninguna figura ideológica disponible. Es precisamente este radicalismo en el inquirir, la inquebrantable disposición para buscar el germen de todo tener sentido en las fuentes mismas de la protofenomenalidad, lo que inserta a la fenomenología en la dignidad y bajo la advocación de la tradición del pensar occidental clásico. Ella da encarnadura a dos movimientos alternativos que en su corresponderse mutuo y radical unicidad conforman su esencia arquitectónica.

La fenomenología es, de entrada, negatividad, pues en un primer momento se manifiesta como recelo, como remisión a comulgar con el sentido hecho; ella se sustrae al imperio del concepto que desde su estatismo designa el mundo como entidad cerrada y ya siempre comprensible. Fenomenología es epojé. La desconfianza, el escepticismo gratuito y sin fundamento con el cual la fenomenología canjea todo peculio en saber heredado constituye su movimiento inaugural. Por él y en él se configura como atavismo salvaje. El pensar de la fenomenología funda en lo sublime ${ }^{6}$, en lo que lo sublime de monstruoso tiene. Como el espíritu hegeliano, éste se hace acreedor de una potencia (Macht) adquirida en el cara a cara con lo negativo, gana su verdad en el desgajamiento (Zerrissenheit). Mas este salvajismo, esta bayoneta calada y presta a desvencijar cualquier tipo de contubernio del saber con la institución, con el bienestar intelectual que en todo momento intenta seducirnos 
y engañarnos al amparo de una apariencia de progreso y linealidad, no es autoafirmación de sí mismo; él abre las puertas a un más allá, a un haber que sólo se moviliza y viene a mostración bajo previa neutralización de toda la masa de saber petrificado que, como los restos inhábiles de un naufragio, constituyen la carga de sentido que a la conciencia natural de cada época le pertenece como propiedad heredada.

El segundo momento tiene un valor de apertura, y pone un contrapunto al primero dándole una extensión positiva. La fenomenología es a la vez reducción. Quiere ir más allá de toda convención, hacerse a un viaje sin retorno, atravesar el umbral de la metafísica y rastrear el sentido de ser en su anonimidad salvaje y sin concepto. En la trayectoria que estos dos movimientos designan se cifra el vigor y la potencia de la fenomenología como atavismo científico. La intuición profunda que al fenomenologizar nos impele es que el ser no hace su discurso a través de la historia de manera neutra. El ser, la esencia del pensar y de lo pensado en el pensar (el ser como lo objetivo del pensar) se enmascara en conceptos, busca subterfugios para dar resguardo a su timidez de principio, para terminar acaso desapareciendo por completo en un olvido que hace del pensar y de la intuición del ser en el pensar un mero trasunto de sí mismos, un simulacro que nos aleja del contacto originario con el pensar en primera persona, del fenómeno como puro fenómeno y nada más que fenómeno. El pensar de la fenomenología no se ocupa con un objeto exterior a ella, sino con la esencia del pensar en cuanto tal, pues rehúsa darse a sí mismo por consabido. Sabe que su ser para sí mismo es pura apariencia y autosatisfacción. En esta tesitura deviene el pensar problema para sí. Se hace sospechoso y repara en que los conceptos operativos de la ontología tradicional que configuran su esencia, desde los cuales piensa y se ejecuta, no son sino odres de viento, sombras y reflejos de una fenomenalidad dimisionaria.

La fenomenología se hace, ya desde Husserl, a la búsqueda de esencias protoontológicas. La fundación de los abstractos nos puede servir muy bien de ejemplo, pues constituye uno de los ejes en paralelo al cual se desarrolla la teoría de la intencionalidad husserliana. Husserl se percata de que la diferencia entre abstracto y concreto es irreducible a cualquier sistema de la lógica. Ambos términos constituyen significados lógicos que nos tropiezan en el análisis de los contenidos en el marco de la fenomenología de la experiencia interna (psicología fenomenológica), y que atañen a todo objeto imaginable. Se trata por lo tanto de contenidos, que antes de toda aprehensión (Auffassung) perceptiva acreditan una tendencia a fenomenalizarse en la percepción como partes abstractas -como por ejemplo el color o la extensión- o bien concretos -singularidades perceptivas apercibidas en una intuición empírica-. Esta diferencia lógica en los contenidos se genera en el marco de las sintesis pasivas de los mismos. La inquietud que este hecho produce, le lleva a Husserl a profundizar en el análisis fenomenológico en busca de evidencias fenomenológicas que den cuenta del origen de los conceptos lógicos.

De este modo viene Husserl, al menos implícitamente, a admitir que la lógica de las significaciones, no está dada de antemano, sino que cada significación lógica cifra, vez a vez, sus aspiraciones en un acto de la intuición, en un cumplimiento en el que la idealidad se da de manera original. La intuición empírica no está, por otra parte, en disposición de servir los objetos eidéticos en su inmediatez, de presentarlos en primera persona. La esencia de esta intuición reside en un mirar tematizante que tan sólo es capaz de hacer visible al objeto en su concretud material empírica. Las teorías clásicas de la abstracción pretendian fundar desde la intuición empírica, recurriendo a explicaciones del más vario pelaje, la posibilidad y el modo de ser propio de los diferentes momentos abstractos (color, extensión, forma, etcétera). Que éstos atañen ineluctablemente a todo contenido es de antemano claro, toda vez que a la diferencia entre parte abstracta y concreto, entre contenido y continente, le cabe un carácter fundatriz y determinante en punto a la fenomenalidad de todo contenido de representación en cuanto tal. Husserl cargará el acento, desde el primer instante, en el peculiar modo de ser de las idealidades, cuyo ser categorial o especifico no se dejará explicitar sino sobrepujando las condiciones de la intuición empírica. La intencionalidad es el elemento llamado a desempeñar la función arquitectónica que permitirá hacer justicia a los objetos específicos en su idealidad de principio, poniendo a la base una intuición de esencias, que, recobrando la tradición aristotélica, vendrá a donar una especie de manera original, en el modo de ser del de suyo (para hablar con Zubiri) o como pulsando en carne propia.

Se podrá decir que la especie o el género categorialmente intuido funda la dependencia de las partes abstractas 
implicadas en un concreto fácticamente experimentado, pero sin incardinarse a él, sin participar como parte constituyente del mismo. El género funda en su idealidad el ser asi de las relaciones a las que toda parte abstracta se ve abocada, pero siempre substrayéndose a él. Visto así es la idealidad la que funda la percepción y no viceversa. El camino de la fenomenología husserliana, cuando no el de la fenomenología toda, describe, empero -y ésta es nuestra tesis-, un movimiento bien distinto al que esta presunción deja insinuar. La percepción, el sentido que en la percepción se va construyendo, es primero en relación a la idealidad. El pensar deja de ser concebido como un elemento heterónomo al campo fenoménico. El pensar se fenomenaliza en el cumplimiento. La mención simbólica, la intención vacía, se fenomenaliza en una intuición que remite a plexos sucesivos de experiencia que se desarroIlan en sistemas teleológicos infinitos. En la reflexión del sentido en la percepción se anuncia la absoluta tendencia del pensar en relación al ser. Esta tendencia se acendra en el movimiento reflexionante (es decir, no determinante) del pensar buscando su cumplimiento, su saturación en lo otro del mundo, haciéndose reflexión teleológica de mundo.

\section{Fenomenología y eXPeriencia}

La fenomenología renuncia a decir de una vez para siempre lo que las cosas son. En su intento por definirlas ella se instala en el punto desde el cual éstas nos interpelan y nos salen al encuentro; es ante todo posibilidad y retorno a las cosas mismas. A diferencia del quehacer científico la fenomenología no aspira a aportar una determinación última del ser de las cosas y del mundo. Ella toma como acontecimiento fundacional y absoluto el haber de un sentido salvaje ${ }^{7}$ o no domesticado que constituye un poro vindicativo a través del cual se realiza la posibilidad misma de acceso al mundo para quienquiera que sea (científico, filósofo o artista). La fenomenología constata el tener sentido de la realidad como un caudal o reducto de sentido bruto y persistente que no se deja reducir al concepto. Analizar el concepto de realidad patrocinado por la fenomenología sería tarea demasiado prolija y excedería definitivamente los propósitos que aquí nos animan. En cualquier caso no sea quizá del todo desatinado señalar una vez más que la idea de realidad que aqui está en juego no se deja entender de ningún modo desde una perspectiva ocupada por la concepción defendida desde la tradición realista que todos conocemos. Desde este punto de vista lo real sería entendido como la posición absoluta de un afuera igualmente absoluto. La fenomenología en cambio entiende lo real como mero correlato, como mención, e incluso como fuga (Husserl no duda en definir la trascendencia de la cosa, su realidad extramental, como una idea en sentido kantiano, susceptible de una determinación en progresión infinita).

Este reducto, este sentido primitivo que constituye nuestra apertura inmediata al mundo es primero con respecto a toda determinación cientificista. En su estar siempre, vez a vez, retornando a la indeterminación esencial del campo fenoménico, a su inclausurabilidad de principio y sin concepto, radica tanto el valor y la posibilidad de su vigencia como la fuerza de su gesto. Las evidencias que la cosa y el mundo acreditan, el sentido que anima su ser en la trascendencia, no germina en ellas sino en virtud del acto de dar sentido, nos dice Husserl en las Investigaciones lógicas. La evidencia que quepa asignarle a la trascendencia brota de actos concretos del significar. El significado o el sentido que se hace evidente a través del cumplimiento en la intuición no se deja hipostatizar en un sistema ideal o en una gramática pura de una vez para siempre, sino que remite, como su condición, a la posibilidad de efectuarse de nuevo, sin solución de continuidad, en la intuición, único lugar donde las significaciones son susceptibles de realizar su significatividad, de hacer visible su sentido o de fenomenalizar el sentido del pensar en cuanto tal. El significado se realiza en el cumplimiento. El decir con sentido remite a actos que se ejecutan bajo el modo de la evidencia intuitiva. La fenomenología es contemporánea de la mirada que descubre el mundo, aspira a, quiere ser ingenuidad.

El decir de la ciencia, por el contrario, acredita un carácter eminentemente dogmático. Se trata de un decir que se agota en el concepto, al que la ciencia imputa la esencia y el tener sentido del pesar en cuanto tales. Los conceptos son entendidos por la ciencia en su calidad de términos (en esta palabra escuchamos lo que en ella hay de acotación, de "Bestimmtheit"), los cuales extirpan a la palabra vivida lo que en ella siempre queda por experimentar, despojándola, por ende, de su indeterminabilidad esencial en virtud de una sobredeterminación categorial. Este estrato de significaciones ya listas y prestas son se- 
dimentos que, sistemáticamente (como elemento en el marco de una institución científica) o asistemáticamente (adoptado la forma de una cosmovisión histórica, de una Weltanschauung) se adhieren al sentido en formación y son movilizadas por él, conformando precipitados opacos o pliegues de significado ya siempre disponibles. El sedimento científico es la sustancia de la que está hecha el concepto, en cuyos auspicios el pensar acredita de antemano un exceso de sobredeterminación proveniente de la institución simbólica.

La ciencia nos hace caer víctimas de una ilusión trascendental, nos compele a soñar con un lenguaje -teoría desde siempre ejercitable y ya encontrado- como simulacro. La trascendencia emerge en la mirada tamatizante del científico como un objeto clausurado sobre sí mismo, un en sí monolítico y sin fisuras, que solamente la teoria -lenguaje del ser y de lo pensado en el pensar- podrá venir a reconstruir a partir de una serie de operaciones intelectuales consideradas en su aislamiento metodológico, sin que se pueda saber muy bien qué tipo de relación media entre mundo en sí y teoría. La ciencia da cuenta del mundo cual si se tratase de un inmenso laboratorio apropiado a su experimentar, sin reparar siquiera que todo teorizar es teorizar en el mundo y desde el mundo.

El fundamento de la lógica no puede ser de orden psicológico, del mismo modo que el fundamento ontológico del objeto visto tampoco puede ser fisiológico. Así como mi ver una cosa determinada no se deja reducir a los fantasmas visuales que mi ojo produce, igualmente la idealidad del objeto lógico no se deja reducir al acto psicológico sobre el que funda la operación matemática. Tanto el ver como el pensar son operaciones de suyo no aislables, incardinadas en la objetividad de un horizonte de mundo que abarca y aporta consistencia ontológica a toda operación, sea su indole la que fuere.

Frente a esta situación la fenomenología quiere ser ante todo fidelidad y cercanía. El fenomenólogo reconoce en la trascendencia su estar dotada de sentido; ésta tiene un sentido desde el cual se articula y se ofrece a la mirada. La fenomenología es, ciertamente, un quehacer teórico. Éste, sin embargo, es considerado como fenómeno de mundo. El decir fenomenológico brota, ya desde sus inicios en Husserl, de la estructura misma del mundo percibido y de la experiencia, en fundamental coalescencia con ellos.
Teoría es ante todo experiencia teórica. Por ello mismo es la fenomenología contemporaneidad; ella pretende habitar el mundo en los diferentes registros del tener sentido desde el cual éste nos interpela, buscando la posibilidad de la teoría en la experiencia y desde la experiencia, sin atreverse a anteponerse a ésta. La conciencia de la que la fenomenología habla no construye el mundo a despecho del mundo.

En su estar ahí fantaseando, dudando, enjuiciando; habitando el mundo, haciéndose sujeto del mundo lo va constituyendo en su en sí. Conciencia es exterioridad. Y viceversa. No hay mundo pensable que no sea mundo de un sujeto y para él. Mundo es interioridad. Es a través de la cosa como yo entro, como por escotillón, en la esfera privada del otro. El otro como sujeto de un mundo que nos es común. Porque el otro es sujeto de un mundo, de un más acá (un más acá que termina siendo mi más acá también), yo puedo comprenderle en el mundo y a través del mundo (Merleau-Ponty). El sentimiento de fruición que una melodía me suscita porta consigo un vínculo intencional. El sentimiento es mención, acto intencional, hace meta en un objeto intencional. La conciencia no se entiende sino en el mundo y partir del mundo.

Muchos equivocos se han agolpado en torno al sentido que a la constitución fenomenológica quepa adscribirle -al menos a aquella de abolengo husserliano. Constitución no es construcción en el sentido en el cual pueda serlo una operación intelectual aislada del resultado que en ella y a través de ella germina. La constitución da expresión a una experiencia de orden trascendental que no pretende dar sino razón de la fenomenalidad del fenómeno en cuanto tal, fijando eidéticamente las estructuras que lo soportan. La fenomenología pretende ser una puesta en escena de la trascendencia en punto al sentido que la atraviesa y jerarquiza.

El descubrimiento de la intencionalidad le permitirá a Husserl arribar a una nueva concepción de la subjetividad. Subjetividad y mundo serán para él como los Dioscuros de los que hablará Ortega. Objeto y conciencia, nóesis y nóema, al igual que Castor y Pólux, son entidades dependientes que no pueden serse la uno sin la otra. En esta heterogracilidad de la conciencia radica en buena medida la gran innovación husserliana, y le separa definitivamente del idealismo de corte clásico. Para Fichte el acto de autoposición de la conciencia es fundacional, es Urtathandlug. 
En Hegel las sintesis omniabarcantes que se despliegan en movimiento dialéctico no tienen por fin otra cosa más que afirmar la autoconciencia como lo absoluto al final del proceso. La conciencia de Husserl no necesita representarse a sí misma, no necesita devenir en objeto de sí misma, inaugurar una relación intencional consigo misma de resultas de la cual ella se ponga a sí como lo absoluto. No es lo absoluto por ser objeto de absoluta autoposición, sino por ser lo absolutamente ponente o sujeto de mundo en grado sumo. Para Husserl el ser absoluto de la conciencia no proviene de su autoponerse, de su automencionarse, sino por ser aquello que en todo momento menciona (das Meinende), por ser intención no intencionada o sujeto incondicionado (Weltverwirklichendes Leben). La conciencia es sobre todo explicitación o mención. Hay mundo, hay conciencia.

Husserl parte de la evidencia de que una misma intuición empírica puede dar cumplimiento (Erfüllung) a una pluralidad de sentidos. La conciencia habita (dahinlebt) el mundo en diferentes modos. Yo puedo levantar la mirada del papel sobre el que ahora mismo escribo. La imagen que se me reofrece es la siguiente: una habitación en semipenumbra, al fondo una ventana, la cual, acaso, regale una hermosa perspectiva sobre un parque. La imagen sensible en cuanto tal puede permanecer invariablemente fija durante un intervalo de tiempo " $X " \mathrm{Y}$, sin embargo, el sentido con el cual es animada puede experimentar drásticas modificaciones. En un primer momento la mención acaso se dirija a la imagen que el cristal me devuelve de mí mismo; evidentemente la ventana e incluso el jardín siguen a la vista, ofreciendo sostén a mi mirada con su densidad inexpugnable, pero permanecen eclipsados toda vez que mi interés perceptivo se centra sobre mi propia imagen espejada. Acto seguido la mención representativa con la cual la imagen sensible es comprendida hace meta en el parque; dejo de vivir en la imagen que el espejo me devuelve, que queda neutralizada. Mi imagen no ha dejado ni un instante de espejarse, mas el rayo de la mirada, a despecho de su resistencia, la atraviesa y hace meta en el parque mismo. Por último cabe aún la posibilidad de que la predilección de la mención perceptiva se dirija al cristal mismo, que de este modo deviene objeto preferente de mi atención perceptiva; sólo él es significado en la percepción. El cristal deja de ser límpido a mi mirada y se afirma como objeto preferente de mi campo visual.

\section{NOTAS}

Recibido: 12 de diciembre de 2007

Aceptado: 15 de enero de 2008
1 El término fue acuñado por Eugen Fink en la VI Meditación Cartesiana. Con él se quiere, en resumidas cuentas, dar a entender que no hay fenomenología posible sin fenomenologizar, esto es, sin intervención de una subjetividad fenomenologizante.

2 Como es sabido en el trabajo comunitario, en el Zusammenarbeit, cifraba Husserl buena parte de sus esperanzas para la nueva ciencia.

3 El termino procede de Merleau-Ponty.

4 Ubiestesia es, recordémoslo una vez más, la traducción que el profesor Antonio Zirión propone en castellano para la palabra alemana Empfindniss.

5 Quizá nadie como el hombre griego haya sido capaz de poner de ma- nifiesto esta relación entre política y fenomenalidad, de ver la política como expresión esencial de la libertad fenomenológica.

6 Entendemos aquí lo sublime no ya en sentido kantiano, como viene siendo usual, sino en cierta connivencia con Hegel. El pensar mira a la negatividad sin ponerse al resguardo, sin sustraerse al peligro de perecer en ella, haciendo estancia en ella.

7 Como bien es sabido el origen esta expresión remite a la filosofía de Merleau-Ponty. No sólo en él, sino ya en Husserl se puede hablar del encuentro con un sentido en estado bruto, de una intencionalidad ejecutiva (fungirende Intentionalität) que da cuenta de las esencias en su estado salvaje. 